\title{
Quantitative interferon-gamma responses predict future disease progression in badgers naturally infected with Mycobacterium bovis
}

\author{
S. N. BUZDUGAN ${ }^{1}$, M. A. CHAMBERS ${ }^{2}$, R. J. DELAHAY ${ }^{3}$ AND J. A. DREWE ${ }^{1 *}$ \\ ${ }^{1}$ Veterinary Epidemiology, Economics and Public Health Group, Royal Veterinary College, London, UK \\ ${ }^{2}$ Animal and Plant Health Agency, Weybridge, UK \\ ${ }^{3}$ National Wildlife Management Centre, Animal and Plant Health Agency, Woodchester Park, \\ Gloucestershire, UK
}

Received 17 October 2016; Final revision 9 August 2017; Accepted 11 August 2017; first published online 11 October 2017

\section{SUMMARY}

The diagnosis and control of Mycobacterium bovis infection (bovine tuberculosis: TB) continues to present huge challenges to the British cattle industry. A clearer understanding of the magnitude and duration of immune response to $M$. bovis infection in the European badger (Meles meles) - a wildlife maintenance host - may assist with the future development of diagnostic tests, and vaccination and disease management strategies. Here, we analyse 5280 diagnostic test results from 550 live wild badgers from a naturally-infected population to investigate whether one diagnostic test (a gamma interferon release [IFN $\gamma$ ] assay, $n=550$ tests) could be used to predict future positive results on two other tests for the same disease (a serological test $[n=2342$ tests $]$ and mycobacterial culture $[n=2388$ tests $]$ ) and hence act as an indicator of likely bacterial excretion or disease progression. Badgers with the highest IFN $\gamma$ optical density (OD) values were most likely to subsequently test positive on both serological and culture tests, and this effect was detectable for up to 24 months after the IFN $\gamma$ test. Furthermore, the higher the original IFN $\gamma$ OD value, the greater the chance that a badger would subsequently test positive using serology. Relationships between IFN $\gamma$ titres and mycobacterial culture results from different types of clinical sample suggest that the route of infection may affect the magnitude of immune response in badgers. These findings identify further value in the IFN $\gamma$ test as a useful research tool, as it may help us to target studies at animals and groups that are most likely to succumb to more progressive disease.

Key words: Diagnosis, epidemiology, immunology, tuberculosis (TB), wildlife.

\section{INTRODUCTION}

The diagnosis and control of Mycobacterium bovis infection (bovine tuberculosis: $\mathrm{TB}$ ) continues to present huge challenges to the British cattle industry [1].

\footnotetext{
* Author for correspondence: J. A. Drewe, Veterinary Epidemiology, Economics and Public Health Group, Royal Veterinary College, Hawkshead Lane, North Mymms, Hertfordshire AL9 7TA, UK. (Email: jdrewe@rvc.ac.uk)
}

The problem is compounded by the presence of $M$. bovis infection in European badgers (Meles meles) which, in addition to cattle, can act as maintenance hosts. A clearer understanding of the magnitude and duration of immune response to $M$. bovis infection in badgers may aid in disease control, for example by informing the development of vaccines and diagnostic tests [reviewed in 2]. For example, cytokines such as gamma interferon (IFN $\gamma$ ) released from activated $\mathrm{T}$ cells, appear to be useful diagnostic and prognostic 
tools in humans and other animals [3-5]. Information on the magnitude and duration of immune responses to $M$. bovis infection in badgers may aid development of management strategies for this disease.

A recent study found that the magnitude of early IFN $\gamma$ responses in badgers naturally infected with M. bovis was positively correlated with a likelihood of subsequent disease progression [2]. However, that analysis was based on a small sample size (56 badgers) and so the generality of their conclusions is uncertain. Here, we use a much larger sample size ( $>500$ badgers) to investigate the representativeness of Tomlinson et al.'s [2] results on a wider scale.

Badgers may become infected with $M$. bovis through a variety of routes including inhalation, bitewounding and, potentially, ingestion [6]. Tomlinson et al. [2] hypothesised that the route of infection may influence the magnitude of the IFN $\gamma$ response and subsequent disease progression, but they had insufficient data to investigate this. Previous studies have suggested that badgers infected through biting may subsequently experience particularity aggressive pathology [7,8] and experimental intradermal injection of $M$. bovis has been linked to progressive systemic infection [9]. Seropositivity is also more likely in situations of progressed disease which suggests that while not directly measuring infectiousness, a positive serological test result may indicate a greater likelihood that this is the case [10]. In the present study, our rich dataset allowed us to examine for relationships between the locations of positive M. bovis culture results (from specific lesions or body areas, which may reflect the route of infection or excretion) and IFN $\gamma$ responses. For bite wounds, this provides insight into how the route of infection may affect the magnitude of the immune response to $M$. bovis infection in badgers.

The duration of immune response is also important. Tomlinson et al. [2] showed that the magnitude of IFN $\gamma$ responses to infection in badgers declined over time, but they did not investigate how soon an animal is likely to become infectious after the first IFN $\gamma$ test. We were able to do so in the present study by focusing on short-term associations (up to 24 months). This revealed insights into the differential timing of the immune responses, which is likely to be particularly useful from an operational perspective, because disease management programmes typically operate over these sorts of timeframes [11].

We hypothesise that, in a badger population naturally infected with $M$. bovis, individuals producing the highest IFN $\gamma$ titres will be the ones most likely to subsequently test positive using $\mathrm{TB}$ tests that measure other arms of the immune system (the humoral response) or detect the pathogen itself (mycobacterial culture). Should this be the case, then it may be possible to use IFN $\gamma$ test results as an indicator of likely future disease progression.

\section{MATERIALS AND METHODS}

\section{Ethics statement}

Badger capture and sampling was carried out under licences from Natural England and the UK Home Office. The protocols were approved by local ethical review within the Food and Environment Research Agency and the Animal Health and Veterinary Laboratories Agency (now the Animal and Plant Health Agency).

\section{Study site and sample collection}

Samples and data were collected from July 2006 to October 2013 from a population of wild badgers living in Woodchester Park, an area of south-west England, which is the focus of a long-term study into badger ecology and TB epidemiology [12]. Badgers were captured, anaesthetised and sampled using wellestablished methods [13] with each badger social group being trapped four times per year, resulting in repeated observations of the same individuals throughout the study period. Trapping was suspended between 1 February and 30 April inclusive when most cubs are very young, confined to the sett, and/or totally dependent on their mother [14]. During January (and, weather dependent, during December and May), when some females may be lactating, traps were checked during the night, and females deemed to be lactating or pregnant on the basis of cursory examination, were released immediately without sampling.

On first capture each badger was given a unique alpha-numeric tattoo, which allowed individuals to be identified thereafter [15]. The location, sex, body weight and condition, reproductive status and age class (cub $[<1 \mathrm{y}]$ or adult $[1 \mathrm{y}+]$ ) of each animal was recorded. The following samples were collected for mycobacterial culture: faeces, urine, tracheal aspirate, oesophageal aspirate, swabs of bite wounds (where present) and swabs of suppurating submandibular lymph node lesions (where present). Bite wounds 
and suppurating submandibular lymph nodes were sampled separately because they are likely to represent different routes of infection. Up to $12 \mathrm{ml}$ of jugular blood was taken for serology and IFN $\gamma$ testing. After recovery from anaesthesia, badgers were released at the point of capture.

Three diagnostic tests were conducted: the IFN $\gamma$ test; the Stat-Pak serological test; and mycobacterial culture of clinical samples (for details of all three tests see reference [16]). Briefly, the IFN $\gamma$ test quantified the secretion of the cytokine IFN $\gamma$ by T-cells following stimulation with purified protein derivatives of bovine (PPD-B) and avian (PPD-A) tuberculin [4]. Results from the IFN $\gamma$ test were available on a continuous scale as optical density (OD) readings of IFN $\gamma$ production. The Stat-Pak (Chembio Diagnostic Systems, New York) identified antibodies produced in response to specific antigens associated with M. bovis [10], giving a binary (positive or negative) test result. The third test was the mycobacterial culture of clinical samples [17] with a positive result recorded for any sample from which $M$. bovis was isolated. All three tests (IFN $\gamma$, Stat-Pak and culture) were conducted on each badger every time it was trapped, except on $2 \%$ of occasions when an insufficient volume of blood was available to allow Stat-Pak or culture to be run. Estimates of the sensitivity and specificity of each of these three tests have been reported separately [18].

\section{Data description and analysis}

Data included IFN $\gamma$, Stat-Pak and culture test results on 550 captured badgers. Animals were enrolled in the study on the date of their first IFN $\gamma$ test (usually the first time they were sampled within the study period) and were followed until the date of their last Stat-Pak or culture test during the period of study. This resulted in a median total observation period per badger of 10 months (range: 1 day to 86 months $(7.2$ years) per badger). Badgers with an observation period of 1 day were trapped and tested just once: therefore a true follow-up time period was not recorded for these animals. However, it was considered possible that their infection status might have been different to those that were trapped more than once (e.g. badgers that were lost to follow-up may have been more likely to have advanced infection) and so to reduce exclusion bias resulting from their omission, the test results of these badgers were included in the analysis by artificially increasing the time period between IFN $\gamma$ and subsequent tests by 1 day.
Table 1. Distribution and categorisation of optical density (OD) values from gamma interferon (IFN $\gamma$ ) test results conducted on the first blood sample collected from each of 550 live badgers trapped at Woodchester Park from July 2006 to October 2013

\begin{tabular}{llll}
\hline \hline $\begin{array}{l}\text { IFN } \gamma \\
\text { category }\end{array}$ & $\begin{array}{l}\text { IFN } \gamma \text { OD values } \\
\text { (PPD-B minus } \\
\text { PPD-A) }\end{array}$ & $\begin{array}{l}\text { Number of } \\
\text { observations }\end{array}$ & $\begin{array}{l}\text { Percentage of } \\
\text { observations }\end{array}$ \\
\hline 0 & $<0$ & 181 & 33 \\
1 & $0 \cdot 000-0.043$ & 277 & 50 \\
2 & $0 \cdot 044-0 \cdot 366$ & 66 & 12 \\
3 & $0 \cdot 367-0.696$ & 15 & 3 \\
4 & $0 \cdot 697-1.920$ & 11 & 2 \\
Total & $0 \cdot 000-1.920$ & 550 & 100 \\
\hline \hline
\end{tabular}

PPD, purified protein derivative; B, bovine; A, avian.

The 'risk factor' of interest included as the explanatory variable in the model was the IFN $\gamma$ titre at the first time each badger was tested. The IFN $\gamma$ titre for each badger was calculated as the quantity of IFN $\gamma$ produced following stimulation with bovine tuberculin purified protein derivative (PPD-B) minus the amount of IFN $\gamma$ produced by stimulation with avian tuberculin purified protein derivative (PPD-A) [4]. Continuous IFN $\gamma$ OD values were used to produce five categories of IFN $\gamma$ results for analysis (Table 1). Because the distribution of data points was highly right-skewed it was not considered appropriate to simply divide the range of values by the number of categories in order to obtain cut-off values. Therefore, negative values (arising from cases where the OD of PPD-A was higher than that for PPD-B) were coded as category 0 (zero), while values higher than zero but $<0.044$ - the current cut-off value for infection in adult badgers [4] - were coded as category 1. Categories 2 and 3 were equally spaced (starting from $0 \cdot 044$ ) using an interval step of $0 \cdot 33$. The category coded as 4 included values higher than $0 \cdot 70$ with the highest IFN $\gamma$ OD value being 1.92 (Table 1).

\section{Relationship between magnitude of IFN $\gamma$ response and other diagnostic test results}

Associations between the categories of the independent variable (IFN $\gamma$ titre) and the dependent variables (subsequent Stat-Pak and culture results) were initially assessed using $\chi^{2}$-square tests. For this analysis, the results of mycobacterial culture of different clinical samples (e.g., urine, faeces and tracheal aspirate) were pooled into one culture result (positive or 
negative) per badger per trapping event. A Cox proportional hazards regression analysis was performed to estimate the rates (probabilities) of subsequent positive Stat-Pak or culture results relative to the different categories of initial IFN $\gamma$ titre. Survival analysis was chosen because this method focuses on 'time-toevent' which permits the calculation of rate ratios. For each badger, the time intervals that elapsed between the first IFN $\gamma$ test and subsequent other TB tests (Stat-Pak or culture) were modelled, to determine whether values of IFN $\gamma$ can be used as a measure of the likelihood of progression of infection. KaplanMeier and Nelson-Aalen curves were plotted for visual assessment of data distribution and to check if the proportional hazards assumption was upheld. Data were formally assessed using a plot of $-\log$ $(-\log )$ survival lines and a Schoenfeld residuals test [19], which revealed that the proportional hazards assumption was not met. Therefore, data were corrected by splitting the observation time over the first year into 3-month intervals (Table S1 in Supplementary Material). A Schoenfeld test indicated that following this step the data no longer violated the proportional hazards assumption. When fitting the Cox regression model to the data, the clustering of multiple observations per badger was specified. This analysis was performed using Stata version 11.2 (Statacorp LP, College Station, Texas, USA). Final models were checked for goodness of fit by using Cox-Snell residuals [20]. The hazard function followed approximately the $45^{\circ}$ line and was exponentially distributed with a hazard ratio that approximated one. Therefore it was concluded that the data fitted the models adequately.

Estimates of rate ratios (the relative probabilities of subsequently obtaining a positive Stat-Pak or culture result following a given IFN $\gamma$ result) were produced for inter-test periods of up to 3, 6, 9 and 12 months, and for the period between 12 and 24 months. For time periods greater than 12 months, annual time categories were used (1-2 years, 2-3 years, etc.) until the end of the study (up to just over 7 years). For follow-up periods in excess of 24 months, the proportional hazards assumption was violated for both Stat-Pak and culture tests. Consequently, only observations made within 24 months of each badger's first IFN $\gamma$ result were included in subsequent analyses. A log-rank test was used to assess equality in survival function between categories of IFN $\gamma$ to determine whether the differences in survival between groups were more than would be expected by chance alone [21].
Analyses were conducted to investigate whether age class or sex confounded or modified the predictive effect of IFN $\gamma$ category on subsequent Stat-Pak or culture test results, and whether the predictive ability of IFN $\gamma$ category significantly differed between these categories of age and sex.

\section{Relationship between IFN $\gamma$ titres and culture results from different types of clinical sample}

We looked for relationships between the mycobacterial culture test results from each type of sample (some of which may be considered as potential proxies for the route of infection: for example: a positive culture of a bite wound swab was taken to indicate that infection had occurred through being bitten) and the IFN $\gamma$ response in the same animal. Mixed effects linear regression models were used, with individual animals as a random effect to avoid pseudo-replication, using data from badgers that were tested by both culture and IFN $\gamma$ test on the same trapping occasions (median of three occasions per badger, range: 1-21). IFN $\gamma$ titres (OD values on a continuous scale) were the response variable, while individual culture sample test results (positive or negative) were the explanatory variables in the model. The IFN $\gamma$ test results were $\log$ transformed to meet the assumption of Normal distribution of regression models' residuals. Any IFN $\gamma$ test results below zero (indicating a higher titre with PPD-A stimulation than with PPD-B stimulation) were considered inconclusive and were removed from the analysis.

\section{RESULTS}

\section{Summary of data and associations}

The majority of badgers $(403 / 550,73 \%)$ had multiple serological (Stat-Pak) and culture test results. There were 2342 Stat-Pak results (median: 3 per badger, range: 1-21) and 2388 'sets' of mycobacterial culture results (median: 3 per badger, range: 1-21). A 'set' of culture results related to the suite of different samples collected from the same badger on the same sampling occasion. The distribution of Stat-Pak and culture test results by category of IFN $\gamma$ titre can be found in Table S2. Each of the 550 badgers contributed one IFN $\gamma$ test result because the 'risk factor' of interest was the IFN $\gamma$ titre at first capture. At the time of this IFN $\gamma$ test, 78 badgers tested positive on Stat-Pak and eight badgers tested positive on culture. 
Table 2. Relative incidence of positive Stat-Pak test results in badgers in relation to previous IFNy titre results over varying time periods

\begin{tabular}{lllllll}
\hline \hline Time period & IFN $\gamma$ category* & Rate ratio & SE & $Z$ & $P>|z|$ & $95 \% \mathrm{CI}$ \\
\hline $0-3$ months & 1 & $3 \cdot 32$ & $1 \cdot 19$ & $3 \cdot 35$ & $0 \cdot 001$ & $1 \cdot 64-6 \cdot 70$ \\
& 2 & $12 \cdot 53$ & $4 \cdot 54$ & $6 \cdot 99$ & $0 \cdot 000$ & $6 \cdot 17-25 \cdot 48$ \\
& 3 & $15 \cdot 19$ & $6 \cdot 86$ & $6 \cdot 03$ & $0 \cdot 000$ & $6 \cdot 27-36 \cdot 83$ \\
& 4 & $13 \cdot 90$ & $6 \cdot 22$ & $5 \cdot 89$ & $0 \cdot 000$ & $5 \cdot 79-33 \cdot 42$ \\
$0-6$ months & 1 & $3 \cdot 00$ & $0 \cdot 94$ & $3 \cdot 53$ & $0 \cdot 000$ & $1 \cdot 63-5 \cdot 54$ \\
& 2 & $12 \cdot 75$ & $3 \cdot 97$ & $8 \cdot 18$ & $0 \cdot 000$ & $6 \cdot 93-23 \cdot 46$ \\
& 3 & $13 \cdot 56$ & $5 \cdot 12$ & $6 \cdot 91$ & $0 \cdot 000$ & $6 \cdot 47-28 \cdot 41$ \\
& 4 & $16 \cdot 33$ & $6 \cdot 63$ & $6 \cdot 88$ & $0 \cdot 000$ & $7 \cdot 37-36 \cdot 18$ \\
$0-9$ months & 1 & $2 \cdot 27$ & $0 \cdot 66$ & $2 \cdot 81$ & $0 \cdot 005$ & $1 \cdot 28-4 \cdot 02$ \\
& 2 & $9 \cdot 14$ & $2 \cdot 72$ & $7 \cdot 43$ & $0 \cdot 000$ & $5 \cdot 10-16 \cdot 38$ \\
& 3 & $10 \cdot 75$ & $3 \cdot 72$ & $6 \cdot 87$ & $0 \cdot 000$ & $5 \cdot 46-21 \cdot 17$ \\
$0-12$ months & 4 & $12 \cdot 13$ & $4 \cdot 81$ & $6 \cdot 30$ & $0 \cdot 000$ & $5 \cdot 58-26 \cdot 38$ \\
& 1 & $1 \cdot 91$ & $0 \cdot 56$ & $2 \cdot 40$ & $0 \cdot 016$ & $1 \cdot 13-3 \cdot 43$ \\
& 2 & $9 \cdot 80$ & $2 \cdot 00$ & $6 \cdot 51$ & $0 \cdot 000$ & $3 \cdot 82-12 \cdot 12$ \\
& 3 & $13 \cdot 77$ & $3 \cdot 66$ & $5 \cdot 44$ & $0 \cdot 000$ & $4 \cdot 09-20 \cdot 00$ \\
$12-24$ months & 4 & $0 \cdot 96$ & $5 \cdot 05$ & $7 \cdot 16$ & $0 \cdot 000$ & $6 \cdot 72-28 \cdot 24$ \\
& 1 & $1 \cdot 18$ & $0 \cdot 29$ & $-0 \cdot 14$ & $0 \cdot 892$ & $0 \cdot 53-1 \cdot 74$ \\
& 2 & $1 \cdot 56$ & $0 \cdot 45$ & $0 \cdot 43$ & $0 \cdot 669$ & $0 \cdot 56-2 \cdot 50$ \\
& 3 & $3 \cdot 14$ & $1 \cdot 69$ & $2 \cdot 16$ & $0 \cdot 034$ & $0 \cdot 60-4 \cdot 08$ \\
\hline \hline
\end{tabular}

Data are derived from five Cox regression models, each of which was run for a different time period (defined as the interval between the IFN $\gamma$ test being conducted and a subsequent Stat-Pak test on the same badger). Rate ratios were calculated by comparing the incidence of positive Stat-Pak test results for badgers in each IFN $\gamma$ category to a baseline rate (category zero in Table 1), which was allowed to vary by time period (proportional hazard assumption). Significant differences from baseline are shaded in grey. As an example, to determine the relative chance of a badger with an IFN $\gamma$ titre of 0.50 subsequently testing Stat-Pak positive 12 months later, compared with a badger with an initial IFN $\gamma$ titre of zero, first determine the category of IFN $\gamma$ using Table 1: in this example it would be category 3 (because the IFN $\gamma$ OD value falls within the range of 0.367-0.696); then look at the rate ratio for this category in time period 0-12 months. The rate ratio of 9.05 can be interpreted as badgers with an IFN $\gamma$ OD value of 0.50 being nine times as likely to test Stat-Pak positive up to a year later than are badgers with an IFN $\gamma$ OD value of zero.

* IFN $\gamma$ categories are detailed in Table 1.

Associations were detected between categories of IFN $\gamma$ and Stat-Pak test results in all time periods examined $\left(\chi^{2}=105 \cdot 7, P<0.001\right)$, and between categories of IFN $\gamma$ and culture test results for observation periods up to 9 months only $\left(\chi^{2}=21, P<0 \cdot 001\right)$. Log-rank tests indicated that the survival function of Stat-Pak test results was not the same for all categories of IFN $\gamma$ in all time periods and for the culture test there was good evidence against equality for a follow-up period of less than a year. This suggests that a difference exists between badgers of different IFN $\gamma$ titres in relation to the probability of subsequently testing positive on Stat-Pak or culture.

\section{Can IFN $\gamma$ be used as a predictor of future Stat-Pak test results?}

The highest rates of Stat-Pak positive test results occurred following the highest IFN $\gamma$ OD values across all time periods (Table 2 ). Predictive ability gradually declined, however, and became inconclusive when follow-up time was more than 12 months (Table 2 and Fig. 1). An exception was for badgers in the highest IFN $\gamma$ category, where the association was sustained over the longest time period (up to 24 months between IFN $\gamma$ and Stat-Pak tests being conducted on the same badger), albeit with a rate ratio of only $3 \cdot 14(95 \% \mathrm{CI}$ : $1 \cdot 09-9 \cdot 02$ ) for this longer period (Table 2 ).

\section{Can IFN $\gamma$ be used as a predictor of future $M$. bovis culture test results?}

IFN $\gamma$ results were generally of less value in predicting future culture test results than they were at predicting subsequent Stat-Pak results. Only badgers with the highest IFN $\gamma$ OD values (category 4) predicted a future positive culture result over every time interval up to 2 years (Table 3). Low numbers of positive culture test results 


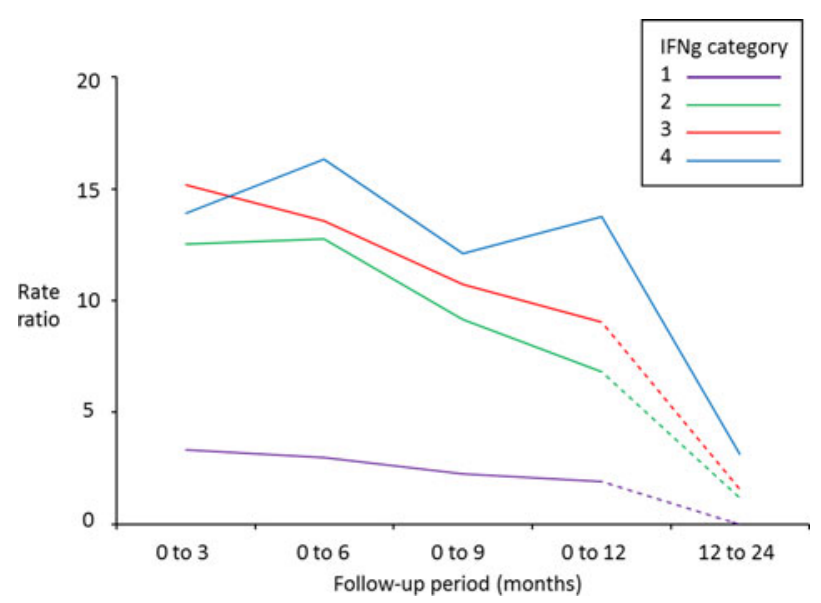

Fig. 1. Rate ratios for subsequently obtaining a positive Stat-Pak test result in badgers after varying follow-up periods, in relation to their initial IFN $\gamma$ titre (category $1=$ lowest IFN $\gamma$ titre; category $4=$ highest IFN $\gamma$ titre: see Table 1 for details of categories) compared with badgers with a negative IFN $\gamma$ titre (category zero). A badger with a rate ratio of 15 can be interpreted as having a 15 times higher chance of testing positive on Stat-Pak within the indicated follow-up period than a badger with a negative IFN $\gamma$ titre. Solid lines indicate significant relationships, and dashed lines indicate relationships that were not found to be significant (see Tables 2 and 3 for $95 \%$ confidence intervals). Data derived from 550 badgers tested with Stat-Pak a total of 2342 times at Woodchester Park from July 2006 to October 2013.

(Table S1), which are likely to reflect the low sensitivity of culture for detecting infected animals [18] explain the wide confidence intervals and why reliable estimates could not be produced for badgers with lower IFN $\gamma$ OD values.

No significant changes in the predictive effects of IFN $\gamma$ categories on subsequent StatPak or culture test results were detected when data were stratified and adjusted for age and sex using the Maentel-Haenszel stratified analysis of rate ratios method. This was true when data were assessed visually - by inspecting the degree of change in rate ratios to examine for confounding, and formally by testing for unequal rate ratios to examine for effect modification. No significant associations were detected between age and sex variables on the rates of positive StatPak or culture results when univariate Cox regression was fitted to study the predictive effect of age and sex explanatory variables. Consequently, age and sex were excluded from the final model.

\section{Comparison with the currently-used IFN $\gamma$ test cut-off level}

Putting these findings in context, the predictive ability of IFN $\gamma$ over one subsequent year can be seen by using as an example badgers with IFN $\gamma$ OD values equal to or greater than 0.044 (the current cut-off for an adult badger to be considered infected [4]). These badgers had at least a six times higher chance of subsequently testing positive on Stat-Pak and culture within 12 months than did animals testing negative (Tables 2 and 3). IFN $\gamma$ results remained associated with other test results 2 years later but the association was less pronounced: badgers with the highest IFN $\gamma$ OD values (category 4: IFN $\gamma$ OD $>0 \cdot 70$ ) had at least a three times higher chance of subsequently testing positive on Stat-Pak, and at least a five times higher chance of subsequently testing positive on culture, than animals with IFN $\gamma$ OD values of zero (Tables 2 and 3).

\section{Relationship between IFN $\gamma$ titres and culture results from different types of clinical sample}

The mixed effects linear regression provided a better fit to the data than a fixed effect linear regression model for both IFN $\gamma$ and culture test results (likelihood ratio test $P<0 \cdot 001$ ). The distribution of IFN $\gamma$ titres varied by $M$. bovis culture result and type of clinical sample (Fig. 2 and Supplementary Figs S1-S6). The likelihood of obtaining a positive $M$. bovis culture result from three types of clinical sample (urine, faeces and bite wound swabs) was positively associated with an increase in IFN $\gamma$ OD value. For example, a positive urine culture result was associated with an IFN $\gamma$ OD value $3 \cdot 5$ times higher than the IFN $\gamma$ OD value associated with a negative urine culture sample $(z$-test $=$ $3.68, P<0 \cdot 001$; 95\% CI: $1 \cdot 8-6 \cdot 9$ ). Similarly, a positive faeces culture result was associated with a 3.5 times increase in IFN $\gamma$ OD value $(z$-test $=3 \cdot 20, P=0 \cdot 001$; $95 \%$ CI: $1 \cdot 6-7 \cdot 4$ ), and a positive bite wound swab culture result was associated with a $3 \cdot 3$ times increase in IFN $\gamma$ OD value $(z$-test $=2 \cdot 85, P=0 \cdot 004 ; 95 \%$ CI: $1 \cdot 4$ 7.7). However, IFN $\gamma$ OD values were not found to be significantly associated with the probability of obtaining any other type of clinical sample (tracheal aspirate, oesophageal aspirate, submandibular lymph node or non-bite-related wounds). It should be noted that the sample sizes for the latter two clinical sample types were very small (samples sizes are given in Table S3).

\section{DISCUSSION}

Our findings indicate that badgers with the highest IFN $\gamma$ titres were those most likely to subsequently test positive on two other types of diagnostic test 
Table 3. Relative incidence of positive M. bovis culture test results in badgers in relation to previous IFNy titre results over varying time periods

\begin{tabular}{lllllll}
\hline \hline Time period & IFN $\gamma$ category* & Rate ratio & SE & $z$ & $P>|z|$ & $95 \%$ CI \\
\hline $0-3$ months & 1 & $1 \cdot 29$ & $1 \cdot 57$ & $0 \cdot 21$ & $0 \cdot 834$ & $0 \cdot 12-14 \cdot 04$ \\
& 2 & $8 \cdot 47$ & $9 \cdot 80$ & $1 \cdot 85$ & $0 \cdot 065$ & $0 \cdot 88-81 \cdot 71$ \\
& 3 & $14 \cdot 60$ & $20 \cdot 76$ & $1 \cdot 88$ & $0 \cdot 060$ & $0 \cdot 90-237 \cdot 22$ \\
& 4 & $15 \cdot 89$ & $22 \cdot 61$ & $1 \cdot 94$ & $0 \cdot 052$ & $0 \cdot 98-258 \cdot 61$ \\
$0-6$ months & 1 & $1 \cdot 37$ & $1 \cdot 69$ & $0 \cdot 26$ & $0 \cdot 796$ & $0 \cdot 12-15 \cdot 26$ \\
& 2 & $12 \cdot 14$ & $13 \cdot 75$ & $2 \cdot 21$ & $0 \cdot 027$ & $1 \cdot 32-111 \cdot 68$ \\
& 3 & $12 \cdot 69$ & $18 \cdot 13$ & $1 \cdot 78$ & $0 \cdot 075$ & $0 \cdot 77-208 \cdot 90$ \\
& 4 & $16 \cdot 60$ & $23 \cdot 66$ & $1 \cdot 97$ & $0 \cdot 049$ & $1 \cdot 02-271 \cdot 18$ \\
$0-9$ months & 1 & $2 \cdot 08$ & $2 \cdot 41$ & $0 \cdot 63$ & $0 \cdot 530$ & $0 \cdot 21-20 \cdot 23$ \\
& 2 & $15 \cdot 68$ & $17 \cdot 27$ & $2 \cdot 50$ & $0 \cdot 012$ & $1 \cdot 81-135 \cdot 70$ \\
& 3 & $16 \cdot 26$ & $23 \cdot 54$ & $1 \cdot 93$ & $0 \cdot 054$ & $0 \cdot 95-277 \cdot 51$ \\
& 4 & $19 \cdot 32$ & $27 \cdot 87$ & $2 \cdot 05$ & $0 \cdot 040$ & $1 \cdot 14-326 \cdot 78$ \\
$0-12$ months & 1 & $2 \cdot 03$ & $1 \cdot 35$ & $1 \cdot 07$ & $0 \cdot 284$ & $0 \cdot 55-7 \cdot 47$ \\
& 2 & $5 \cdot 54$ & $4 \cdot 04$ & $2 \cdot 34$ & $0 \cdot 019$ & $1 \cdot 32-23 \cdot 17$ \\
& 3 & $8 \cdot 15$ & $9 \cdot 68$ & $1 \cdot 77$ & $0 \cdot 077$ & $0 \cdot 80-83 \cdot 49$ \\
& 4 & $12 \cdot 84$ & $14 \cdot 94$ & $2 \cdot 19$ & $0 \cdot 028$ & $1 \cdot 31-125 \cdot 82$ \\
$12-24$ months & 1 & $1 \cdot 03$ & $0 \cdot 67$ & $0 \cdot 05$ & $0 \cdot 959$ & $0 \cdot 30-3 \cdot 61$ \\
& 2 & 0 & 0 & $-63 \cdot 93$ & $0 \cdot 000$ & $0-0$ \\
& 3 & 0 & 0 & $-51 \cdot 75$ & $0 \cdot 000$ & $0-0$ \\
& 4 & $5 \cdot 80$ & $3 \cdot 99$ & $2 \cdot 56$ & $0 \cdot 011$ & $1 \cdot 508-22 \cdot 33$ \\
\hline \hline
\end{tabular}

Data derived from five Cox regression models as described for Table 2. Significant differences from baseline are shaded in grey. For method of interpretation, see legend to Table 2.

* IFN $\gamma$ categories are detailed in Table 1.

(serology and culture) and this effect was detectable for up to 24 months. The Stat-Pak serological test showed a positive trend in its dose-response relationships with IFN $\gamma$, meaning that the higher the original IFN $\gamma$ OD value, the greater the chance that a badger would subsequently test positive on Stat-Pak. The relationship between IFN $\gamma$ and culture was less clear, which may be a real effect or could have been influenced by the low number of culture-positive badgers in the analysis (69/2388 or 3\%). These results concur with and build on those reported by Tomlinson et al. [2] which, due to strict restriction criteria, were based on a substantially smaller dataset (56 badgers, as compared with 550 in the present study). We believe that this positive association between IFN $\gamma$ response and subsequent diagnostic test results indicating disease progression is robust, because two very different analytical approaches - linear modelling [2] and survival analysis (this study) - led to the same conclusion. Further, research in cattle infected with TB has shown the magnitude of the IFN $\gamma$ response (to ESAT-6) to be proportional to disease progression [5]. The present study of badgers adds new evidence supporting the proposition that IFN $\gamma$ appears to be a useful prognostic immunological marker in several species.

We detected no association between a badger's age class (cub vs. adult) or sex and the predictive effect of IFN $\gamma$ category on subsequent StatPak or culture test results. This appears to contrast with previous research [2] where associations were found between the magnitude of IFN $\gamma$ titres and age (lower IFN $\gamma$ responses in cubs) and sex (lower responses in males than females). A key difference in study design may explain this apparent discrepancy: Tomlinson et al. [2] analysed a very small set of data from badgers preselected as positive based on their IFN $\gamma$ test (OD values of $\geqslant 0.044$ for adults and $\geqslant 0.023$ for cubs) whereas the present study analysed a much larger dataset of IFN $\gamma$ test results regardless of whether they were considered positive for infection (i.e. the present analysis included IFN $\gamma$ test OD values of $<0.044$ for adults and $<0.023$ for cubs). This means the present study is likely to have included badgers before they were infected, or at earlier stages of infection.

In their previous study, Tomlinson et al. [2] showed that the magnitude of IFN $\gamma$ responses to infection in 


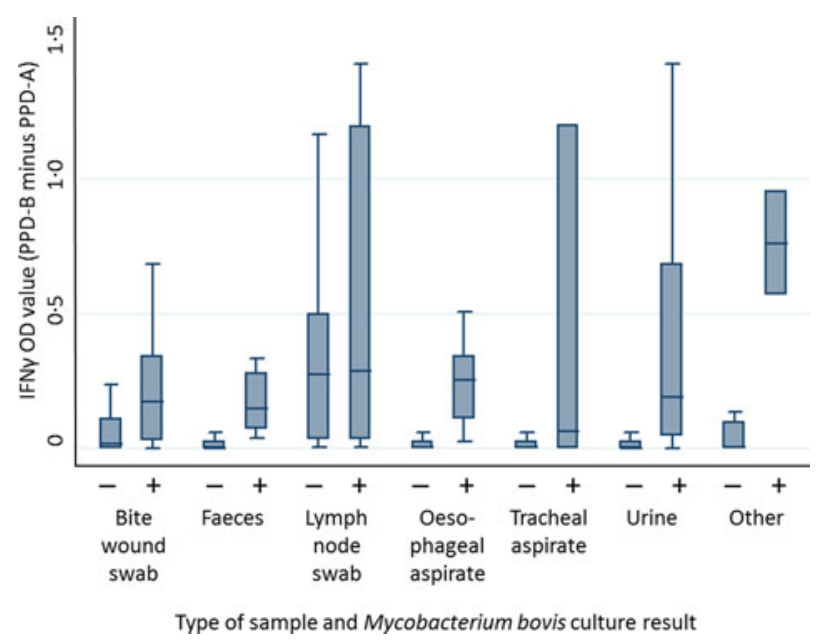

Fig. 2. Distribution of IFN $\gamma$ OD values stratified by mycobacterial culture result across a range of different clinical samples. Data shown includes 2205 observations from 546 badgers. $+=$ positive culture result, $-=$ negative culture result. Outliers are not shown.

badgers declined over time, but they did not investigate how quickly an animal is likely to become infectious. Analysing the larger dataset in the present study allowed us to tease out differential information on the relative rates of positive diagnostic test results, subsequent to the IFN $\gamma$ test, over a range of fairly short time periods (particularly 6, 9, 12, and 24 months). It is less easy to interpret the results for the 0-3 month time period because badgers were rarely caught more frequently than every 3 months (due to trapping occurring four times a year), and hence a high proportion of the positive Stat-Pak and culture test results recorded in this time period occurred at the time of the initial IFN $\gamma$ test. For these badgers, this prevented us from investigating correlations between IFN $\gamma$ titres and disease progression because the available information was limited to the set of diagnostic test results obtained at the time of the IFN $\gamma$ test. This was much less of a problem for time periods longer than 3 months because badgers that contributed data had by then been sampled at least twice. Overall, our findings provide insights into the differential timing of the immune responses, and may enhance the value of the IFN $\gamma$ test as a research tool. Identification of those individuals and groups that may be more likely to experience disease progression may be particularly valuable for field investigations of the behavioural consequences and transmission dynamics of TB in badgers.

Although IFN $\gamma$ test results are generated on a continuous scale (OD values), the diagnosis of infection status in badgers is currently based on whether the OD value falls above or below a pre-determined cutoff $(0.044$ for adult badgers, 0.023 for cubs: ref. [22]). Hence, as the diversity in the range of OD values is not fully used for diagnosis, some information is lost. Results of our analyses indicate that by using the raw OD values it is possible to go beyond answering whether or not an animal is 'positive', and to potentially infer the stage of infection and the likelihood that it will subsequently test positive on other diagnostic tests, within an up to 24-month time window. Those animals producing the highest values of IFN $\gamma$ (i.e. category 4 in the present analysis) were most likely to go on to also test positive on culture (indicating detection of excretion). On the basis of this evidence it would be tempting to apply a cut-off for IFN $\gamma$ OD values of 0.697 (the lower boundary of our category 4) rather than the currently used cut-off of 0.044 in order to identify animals most likely to go on to become infectious. However, our results suggest that the current cut-off is useful, as badgers with an OD value greater than or equal to this cut-off are likely to go on to test positive on Stat-Pak. This is relevant because previous studies indicate that a positive StatPak result is more likely to occur in badgers at advanced stages of TB [10] and seropositivity identifies badgers with the greatest probability of transmitting infection [22]. Moreover, raising the IFN $\gamma$ cut-off on the basis of culture results would likely result in some future excretors being missed because the culture of clinical samples is known to be an insensitive diagnostic approach in live badgers [16]

The finding that increased IFN $\gamma$ OD values were associated with positive culture results from some clinical samples (bite wound swabs, urine and faeces) but not others (tracheal aspirates and oesophageal aspirates) suggests that perhaps the routes of infection (bite wounds) or subsequent dissemination of infection (e.g. to the kidneys giving rise to bacteria in urine) may affect the magnitude of immune response in badgers. This is consistent with evidence from studies of TB pathology in badgers which indicate that disease progression in animals with bite wounds may be rapid [6]. No relationship could be established between tracheal aspirate culture results and IFN $\gamma$ titres because of the low proportion of positive culture results from this type of sample ( $0 \cdot 1 \%$ compared with $15 \%$ for bite wound swabs: Table S3). The relationships between IFN $\gamma$ OD values and both the pathogenesis and expression of TB in badgers are worthy of further research. 
There are some limitations inherent in our analysis, one of which concerns the proportional hazards approach, which assumes that the effect of the predictor variable (the IFN $\gamma$ OD value) was constant for the duration of the study. This is unlikely to have been truly the case, as a badger's IFN $\gamma$ titre is expected to vary over time and with the course of infection [2]. Nevertheless, the assumption was not violated for a follow-up period of 2 years (as indicated by the formal assessment of survival lines and a Schoenfeld residuals test) and hence the analyses and conclusions appear valid. A second limitation was the uneven distribution of observations amongst categories of IFN $\gamma$ response. In order to address the limited number of observations in the highest categories of IFN $\gamma$ (due to few badgers giving very high IFN $\gamma$ OD readings) we focused on interpreting the trends in outputs rather than individual values. The two highest IFN $\gamma$ categories accounted for only approximately $4 \%$ of observations for both Stat-Pak and culture (Table S2), which is likely to have resulted in low statistical power for the parameters estimated. Moreover, the mycobacterial culture test has limited sensitivity in live badgers: as low as $10 \%$ in some cases [16], which means that the true predictive ability of IFN $\gamma$ is very likely to be higher than that described here. Only $3 \%$ of culture test results were positive in comparison with $21 \%$ of Stat-Pak tests, thus any relationship between IFN $\gamma$ and culture may be masked by inaccurate data and/or a low sample size. These limitations could potentially be addressed in future studies by improving the sensitivity of the culture test, possibly by using an extended sampling protocol involving more types of samples or more frequent sampling although this is unlikely to be practical. A more practical alternative would be to repeat the analysis in the future when more data become available.

In conclusion, we have shown that the magnitude of the IFN $\gamma$ response in badgers naturally infected with $M$. bovis is positively associated with the subsequent likelihood of disease progression, reflected in rates of positive results to two different diagnostic tests over a range of time periods. Although this knowledge would be of some value in field operations, for example by helping to identify individual badgers most likely to become infectious to others, the practical requirements for performing the IFN $\gamma$ test - such as the overnight incubation of blood samples and the relatively large volumes required - severely limit its potential applications as a management tool. Nevertheless, measurement of the magnitude of the IFN $\gamma$ response is a useful research tool as it may help us to target studies at animals and groups that are most likely to succumb to more progressive disease.

\section{SUPPLEMENTARY MATERIAL}

The supplementary material for this article can be found at https://doi.org/10.1017/S0950268817001960.

\section{ACKNOWLEDGEMENTS}

This research was funded by the UK Department for Environment, Food and Rural Affairs (project SE3265). RVC manuscript number: PPH 01437. We thank the fieldworkers at the Animal and Plant Health Agency (APHA) who contributed to data collection in Woodchester Park, and staff of the Bacteriology Department of APHA for generating the test results and for technical support. Glyn Hewinson and Martin Vordermeier provided helpful comments on the manuscript.

\section{DECLARATION OF INTEREST}

None.

\section{REFERENCES}

1. Defra. Latest statistics on tuberculosis (TB) in cattle in Great Britain. National Statistics from the Department for Environment, Food \& Rural Affairs and the Animal and Plant Health Agency. 2016 (https://www.gov.uk/government/statistics/incidence-oftuberculosis-tb-in-cattle-in-great-britain). Accessed 14 October 2016.

2. Tomlinson AJ, et al. Association of quantitative interferon- $y$ responses with the progression of naturally acquired Mycobacterium bovis infection in wild European badgers (Meles meles). Immunology 2015; 144: 263-270.

3. Lalvani A, Millington KA. T cells and tuberculosis: beyond interferon- $\gamma$. Journal of Infectious Diseases 2008; 197: 941-943.

4. Dalley D, et al. Development and evaluation of a gamma-interferon assay for tuberculosis in badgers (Meles meles). Tuberculosis (Edinburgh, Scotland) 2008; 88: 235-243.

5. Vordermeier HM, et al. Correlation of ESAT-6-specific gamma interferon production with pathology in cattle following Mycobacterium bovis BCG vaccination against experimental bovine tuberculosis. Infection and Immunity 2002; 70: 3026-3032.

6. Corner LA, et al. The distribution of Mycobacterium bovis infection in naturally infected badgers. Veterinary Journal (London, England: 1997) 2012; 194: 166-172. 
7. Clifton-Hadley RS, Wilesmith JW, Stuart FA. Mycobacterium bovis in the European badger (Meles meles): epidemiological findings in tuberculous badgers from a naturally infected population. Epidemiology and Infection 1993; 111: 9-19.

8. Jenkins HE, et al. The prevalence, distribution and severity of detectable pathological lesions in badgers naturally infected with Mycobacterium bovis. Epidemiology and Infection 2008; 136: 1350-1361.

9. Pritchard DG, et al. Experimental infection of badgers (Meles meles) with Mycobacterium bovis. Epidemiology and Infection 1987; 98: 145-154.

10. Chambers MA, et al. Validation of the BrockTB Stat-Pak assay for detection of tuberculosis in Eurasian badgers (Meles meles) and influence of disease severity on diagnostic accuracy. Journal of Clinical Microbiology 2008; 46: 1498-1500.

11. Defra. Bovine TB: summary of badger control monitoring during 2015. Policy paper from the Department for Environment, Food \& Rural Affairs. 2015 (https://www. gov.uk/government/publications/bovine-tb-summary-ofbadger-control-monitoring-during-2015). Accessed 14 October 2016.

12. Delahay RJ, et al. The spatio-temporal distribution of Mycobacterium bovis (bovine tuberculosis) infection in a high-density badger population. Journal of Animal Ecology 2000; 69: 428-441.

13. Delahay RJ, et al. Long-term temporal trends and estimated transmission rates for Mycobacterium bovis infection in an undisturbed high-density badger (Meles meles) population. Epidemiology and Infection 2013; 141: 1445-1456.

14. Woodroffe R, et al. Welfare of badgers (Meles meles) subjected to culling: development and evaluation of a closed season. Animal Welfare 2005; 14: 19-25.

15. Cheeseman CL, Harris $\mathbf{S}$. Methods of marking badgers (Meles meles). Journal of Zoology 1982; 197: 289-292.

16. Drewe JA, et al. Diagnostic accuracy and optimal use of three tests for tuberculosis in live badgers. PLOS ONE 2010; 5: e11196.

17. Gallagher J, Horwill DM. A selective oleic acid albumin agar medium for the cultivation of Mycobacterium bovis. Journal of Hygiene 1977; 79: 155-160.

18. Buzdugan SN, et al. Diagnosis of tuberculosis in groups of badgers: an exploration of the impact of trapping efficiency, infection prevalence and the use of multiple tests. Epidemiology and Infection 2016; 144: 1717-1727.

19. Grambsch PM, Therneau TM. Proportional hazards tests and diagnostics based on weighted residuals. Biometrika 1994; 81: 515-526.

20. Cox DR, Snell EJ. A general definition of residuals. Journal of the Royal Statistical Society Series B (Methodological) 1968; 30: 248-275.

21. Allison PD. Event History Analysis: Regression for Longitudinal Event Data. Newbury Park, California: Sage Publications, Inc., 1984.

22. Chambers MA, et al. Performance of TB immunodiagnostic tests in Eurasian badgers (Meles meles) of different ages and the influence of duration of infection on serological sensitivity. BMC Veterinary Research 2009; 5: 1-7. 